\title{
The Structure of AGNs from X-Ray Absorption Variability
}

\author{
Guido Risaliti ${ }^{1,2}$ \\ ${ }^{1}$ INAF - Osservatorio Astrofisico di Arcetri, Largo E. Fermi 5, Firenze, Italy \\ Email: risaliti@arcetri.astro.it \\ ${ }^{2}$ Harvard-Smithsonian Center for Astrophysics, 60 Garden Street, Cambridge, MA 02138, USA
}

\begin{abstract}
We present new evidence of X-ray absorption variability on time scales from a few hours to a few days for several nearby bright AGNs. The observed $N_{\mathrm{H}}$ variations imply that the X-ray absorber is made of clouds eclipsing the X-ray source with velocities in excess of $10^{3} \mathrm{~km} \mathrm{~s}^{-1}$, and densities, sizes and distances from the central black hole typical of BLR clouds. We conclude that the variable X-ray absorption is due to the same clouds emitting the broad emission lines in the optical/UV. We then concentrate on the two highest signal-to-noise spectra of eclipses, discovered in two long observations of NGC 1365 and Mrk 766, and we show that the obscuring clouds have a cometary shape, with a high density head followed by a tail with decreasing $N_{\mathrm{H}}$. Our results show that X-ray time resolved spectroscopy can be a powerful way to directly measure the physical and geometrical properties of BLR clouds.
\end{abstract}

Keywords. galaxies: active; galaxies: Seyfert; X-rays: galaxies; X-ray: individual (NGC 1365, Mrk 766)

\section{Introduction}

X-ray absorption variability is a common feature in AGNs. An analysis of a sample of nearby X-ray obscured AGNs with multiple X-ray observations performed a few years ago (Risaliti et al. 2002 ) revealed that column density $\left(N_{\mathrm{H}}\right)$ variations are almost ubiquitous in local Seyfert galaxies. More recent observations performed with XMM-Newton, Chandra, and Suzaku further confirmed this finding. The physical implications of these measurements are that the circumnuclear X-ray absorber (or, at least, one component of it) must be clumpy, and located at sub-parsec distances from the central source. The comparison between different observations, typically performed at intervals of months to years, provides only upper limits to the intrinsic time scales of $N_{\mathrm{H}}$ variations. An improvement of these estimates can be obtained only through observational campaigns within a few days or weeks and/or through the search for $N_{\mathrm{H}}$ variations within single long observations. Such short time-scale studies have been already performed for a handful sources: NGC 1365 (Risaliti et al. 2005, 2007, 2009), NGC 4388 (Elvis et al. 2004), and NGC 4151 (Puccetti et al. 2007). In particular, in the case of the AGN in NGC 1365 we revealed extreme spectral changes, from Compton-thin $\left(N_{\mathrm{H}} \sim 10^{23} \mathrm{~cm}^{-2}\right)$ to reflectiondominated $\left(N_{\mathrm{H}}>10^{24} \mathrm{~cm}^{-2}\right)$ in time scales from a couple of days to $\sim 10$ hours. Such rapid events imply that the absorption is due to clouds with velocity $v>10^{3} \mathrm{~km} \mathrm{~s}^{-1}$, at distances of the order of $10^{4}$ gravitational radii, assuming that they are moving with Keplerian velocity around the central black hole. The physical size and density of the clouds are of the order of $10^{13} \mathrm{~cm}$ and $10^{10}-10^{11} \mathrm{~cm}^{-3}$, respectively. $\dagger$ All these physical parameters are typical of broad-line region (BLR) clouds, strongly suggesting that the

$\dagger$ For a detailed derivation and discussion of these parameters, see Risaliti et al. (2009). A rough estimate is also mentioned here in $\S 2$. 

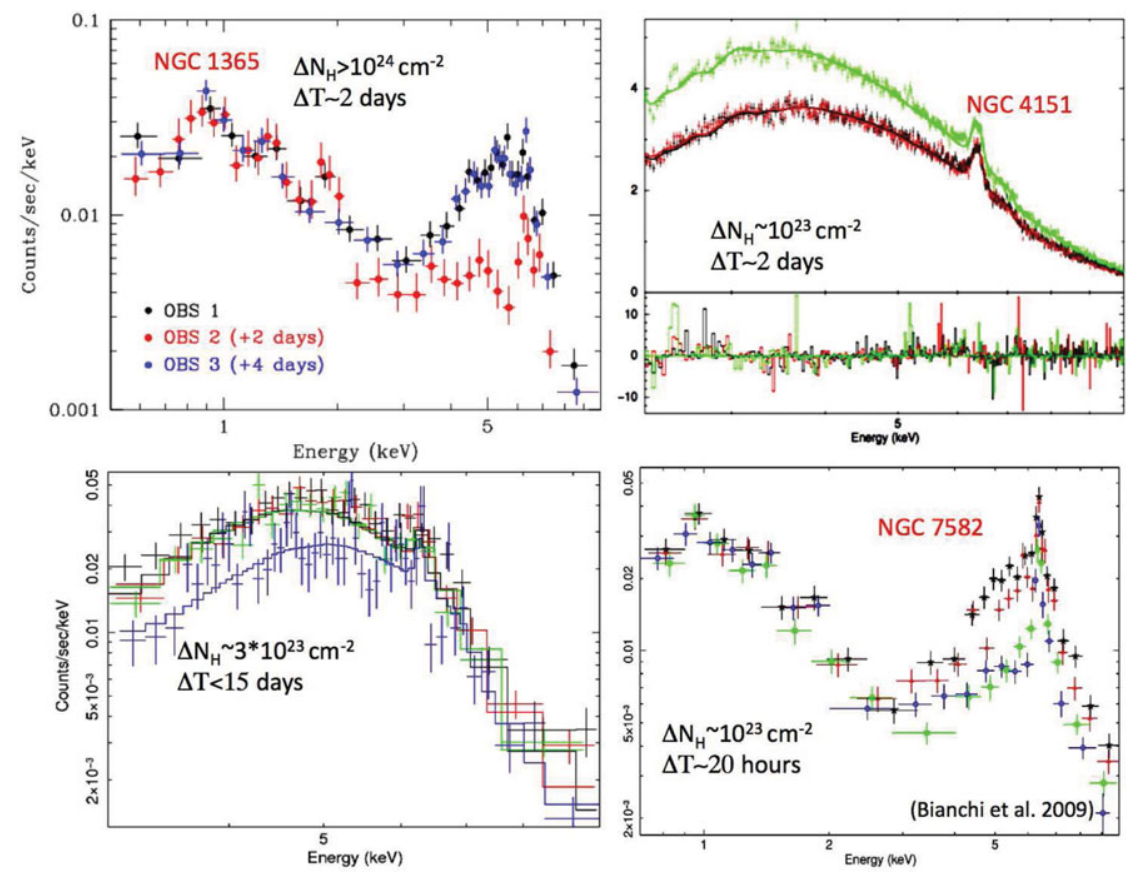

Figure 1. Four examples of new $N_{\mathrm{H}}$ variations on time scales of a few days discovered with campaigns of snapshot observations.

X-ray absorber and the clouds responsible for broad emission lines in the optical/UV are one and the same.

Here we present more evidence, based on time-resolved X-ray spectroscopy, of variable X-ray absorption due to BLR clouds, and we show how in the best cases we can use $\mathrm{X}$-ray observations to directly probe the nature and physical state of BLR clouds.

\section{X-Ray Absorption from BLR Clouds in AGNs}

X-ray absorption due to BLR clouds crossing the line of sight to the observer implies variability time scales from hours to weeks, depending of the physical size of the Xray source. We can obtain a rough estimate of the occultation times assuming a cloud moving with Keplerian velocity around the central black hole, and a size of the X-ray source of $10 R_{\mathrm{G}}: T \sim 3 \times 10^{5} M_{7} v_{3}^{-1}$, where $M_{7}=M /\left(10^{7} M_{\odot}\right)$ and $v_{3}=v /\left(10^{3} \mathrm{~km} \mathrm{~s}^{-1}\right)$. Two observational methods have been used to investigate $N_{\mathrm{H}}$ variations on these time scales:

1. Snapshot observations of the same source, repeated every few days. After the first successful campaign of six short (10 ks) Chandra observations of NGC 1365, we repeated this approach for other bright Compton-thin sources with column densities between $10^{23}$ and $10^{24} \mathrm{~cm}^{-2}$, using both archival data and dedicated observations. In this way we discovered clear cases of $N_{\mathrm{H}}$ variations in NGC 4151 and UGC 4203 (one of the few "changing look" sources, known for having been observed in the past in both Compton-thin and reflection-dominated states). A further case of such variations has been discovered by Bianchi et al. (2009) in NGC 7582 (Figure 1).

2. Study of the spectral variations of bright sources during long observations. This method consists of a two-phase analysis: we first use the hardness-ratio light curve to 

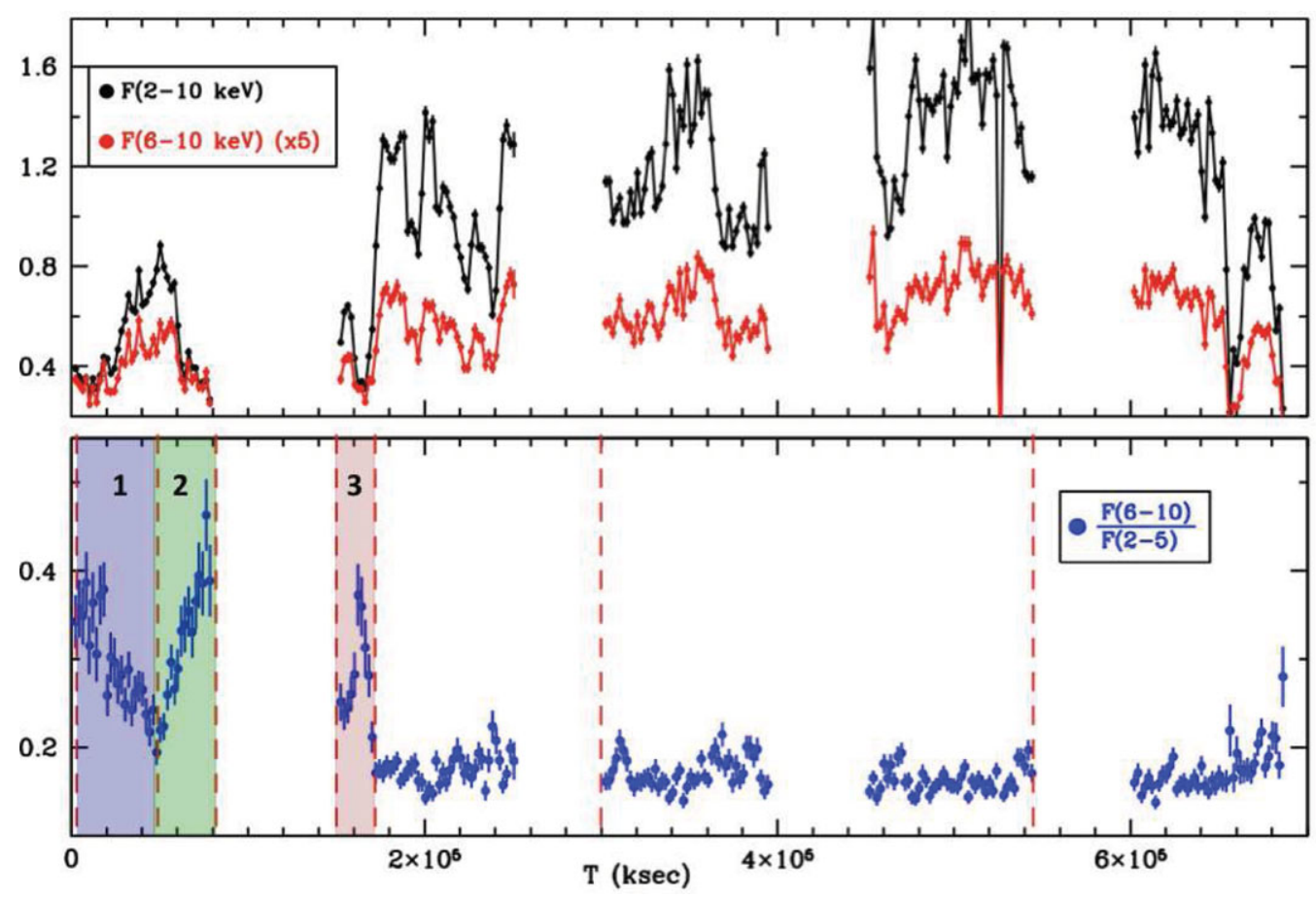

Figure 2. Flux (top) and hardness ratio (bottom) light curves from the $X M M-N e w t o n$ long observation of Mrk 766. The observation is made in five consecutive XMM-Newton orbits.

select the time intervals where strong spectral variations occurred; we then perform a complete analysis of the spectra obtained from these intervals, in order to measure possible $N_{\mathrm{H}}$ varations (and to check if the spectral changes are due to other effects, such as variations of the slope of the continuum emission).

This approach is illustrated in Figure 2 for the long XMM-Newton observation of Mrk 766. A complete analysis of these data is presented by Turner et al. (2007) and Miller et al. (2007). With respect to these works, our analysis is in many aspects less detailed (though the results are in full agreement), but is more effective in isolating the effects of possible $N_{\mathrm{H}}$ variations. We note that Mrk 766 is a narrow-line Seyfert 1 (NLS1), so on average we do not expect to observe complete X-ray absorption of the Xray source. However, as we show below, isolated clouds occasionally cross the line of sight, producing measurable absorption in the X-ray spectrum. The upper panel of Figure 2 shows the standard $2-10 \mathrm{keV}$ flux light curve for this observation, with the well known strong variability on time scales of thousands of seconds, or even shorter. The lower panel shows the light curve of the $(6-10 \mathrm{keV}) /(2-5 \mathrm{keV})$ flux ratio. In general, this light curve shows much smaller variations, indicating that the continuum shape remains the same during most of the luminosity variations. However, clear exceptions are observed in at least three intervals, highlighted in Figure 2. During these intervals it is possible that a cloud with $N_{\mathrm{H}} \sim 10^{23} \mathrm{~cm}^{-2}$ has covered the central source, strongly decreasing the observed flux in the soft band, without affecting the hard band, and therefore increasing the observed hardness ratio.

Following this interpretation, in the first interval we should be observing a cloud uncovering the X-ray source (with the covering phase having occurred before the beginning of the observation); in the second interval another cloud shoud be covering the X-ray 


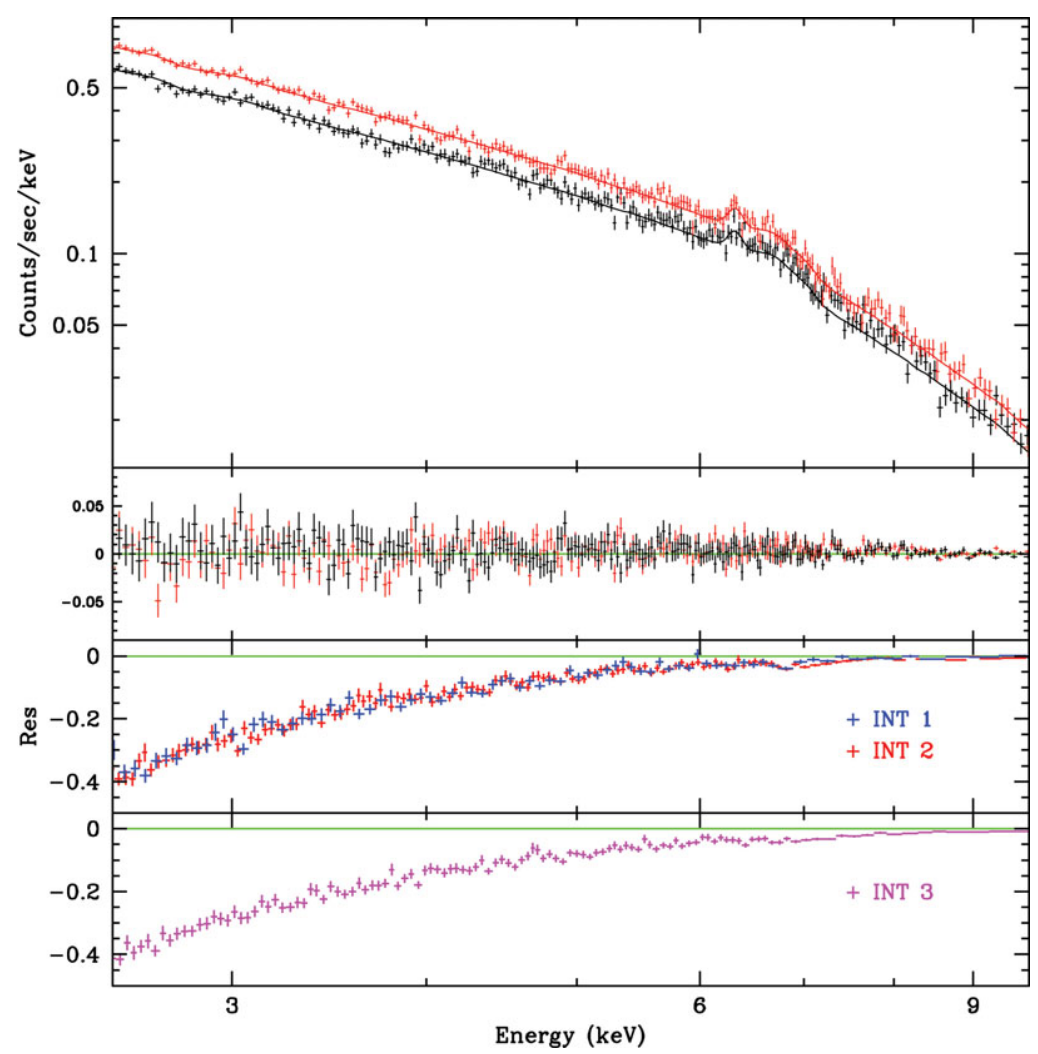

Figure 3. Results from the spectral analysis of the eclipses observed in Mrk 766. Top two panels: spectra, best-fit model and residuals from the third and fourth orbit (Figure 2), where no spectral changes are observed. Bottom panels: difference between the spectra in the three intervals with spectral variations (Figure 2) and the best fit model for the third and fourth orbit.

source, with the uncovering phase not observed due to the "dead time" between two consecutive $X M M-N$ ewton orbits. Finally, in the third interval we should be observing the whole eclipse.

In order to check this scenario, we performed a complete analysis of the spectra obtained from the three highlighted intervals, and of those obtained from the third and fourth orbit, representing the standard spectral state of the source. In this analysis we allowed all the main spectral parameters of the model to vary among the different intervals. The results of this study, illustrated in Figure 3 are (1) the $2-10 \mathrm{keV}$ spectrum obtained from the third and fourth orbit (the "standard" state) which is well-reproduced by a typical model for type 1 AGNs, consisting of a power law, a reflection component, and an iron emission line, and (2) the spectral variations observed in the three intervals discussed above, which are completely reproduced by three absorption components with column densities in the range $1-3 \times 10^{23} \mathrm{~cm}^{-2}$.

The analysis briefly summarized here has been applied to several sources with long archival observations by XMM-Newton and Suzaku. Up to now, we obtained unambiguous evidence for $N_{\mathrm{H}}$ variations in NGC 4388, MCG-6-30-15, Mrk 766, NGC 1365. A further case of similar variations has been found in a Suzaku observation of NGC 3227 (Liu et al. 2010, submitted). 

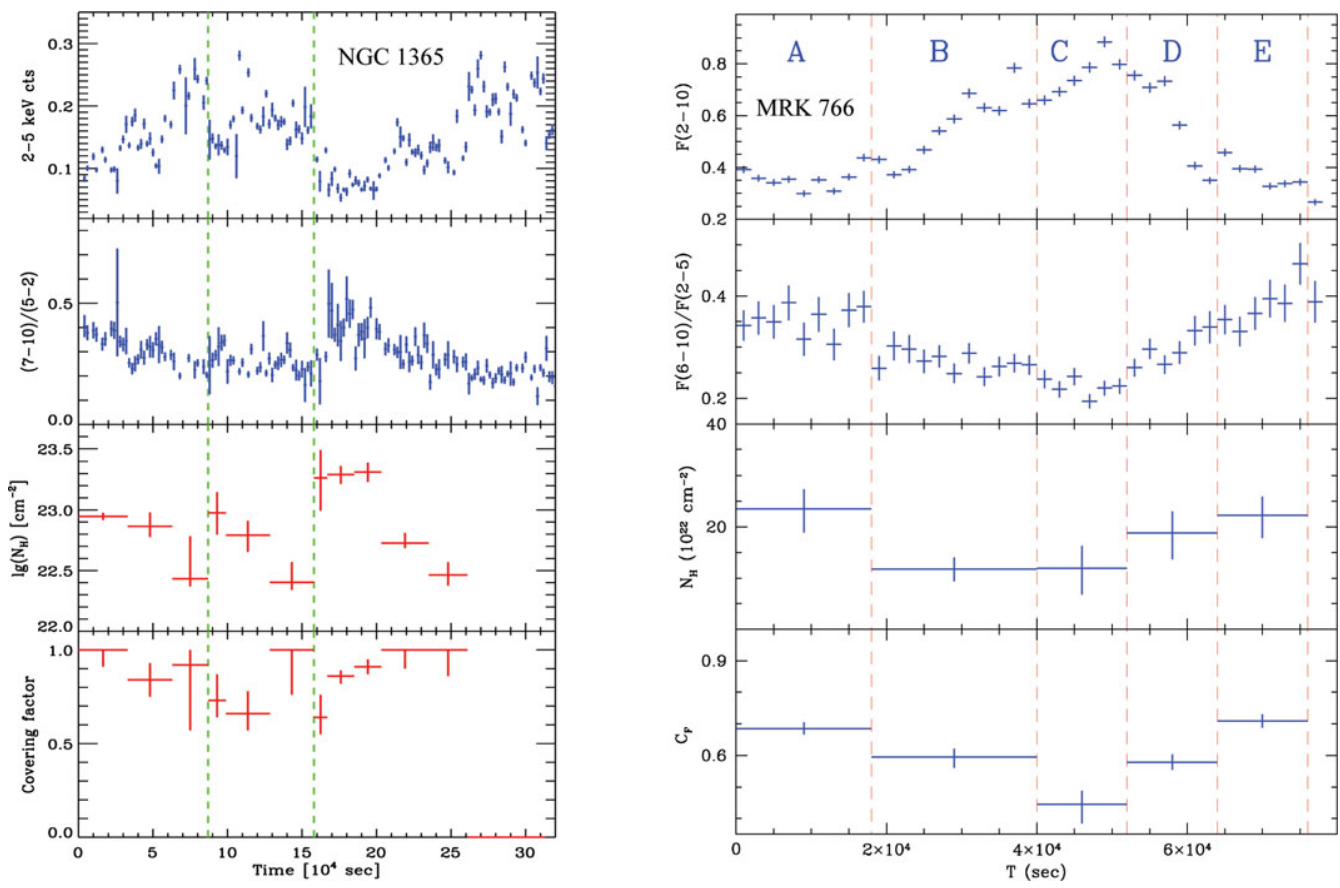

Figure 4. Detailed analysis of a Suzaku observation of NGC 1365 (left) and of the first two intervals of the Mrk 766 observation highlighted in Figure 2 (right). In each panel, we show, from top to bottom, the light curves for flux, hardness ratio (in the case of Mrk 766 these two plots are just a zoom of the light curves shown in Figure 2), $N_{\mathrm{H}}$, and covering factor.

A summary of all the positive detections obtained so far is shown in Table 1. In all cases the inferred physical parameters for the obscuring clouds clearly indicate that the $\mathrm{X}$-ray absorption is due to BLR clouds.

\section{Structure of the BLR Clouds from X-Ray Spectroscopy}

The analysis presented above can be further refined in the few cases where the statistics are high enough to perform a more detailed study, or where multiple occultation events occur. In particular, we briefly summarize two results from the analysis of the two so-far best-studied sources, Mrk 766 and NGC 1365.

Velocity distribution of BLR clouds. The analysis of the spectral variations in Mrk 766 (Figures 2-4) directly provides contraints on the distribution of BLR cloud velocities from the comparison of the durations of the observed occultations. The actual values of the cloud velocity in each case cannot be precisely determined, being dependent on the exact size of the X-ray source. However, the ratio between the duration of the different eclipses is an estimate of the velocity ratios. In particular, the duration of the two first occultation events (Figure 2), is about 4-5 times longer than that of the third eclipse. This suggests a similar spread in the velocity of BLR clouds.

Structure of BLR clouds. The model adopted to reproduce the observed occultation events consists of an absorption component with a constant (during the eclipse) $N_{\mathrm{H}}$, and a variable covering fraction, $C_{F}$. This is an extreme simplification of the cloud structure, which is assumed to have constant column density and very sharp edges. The model can 

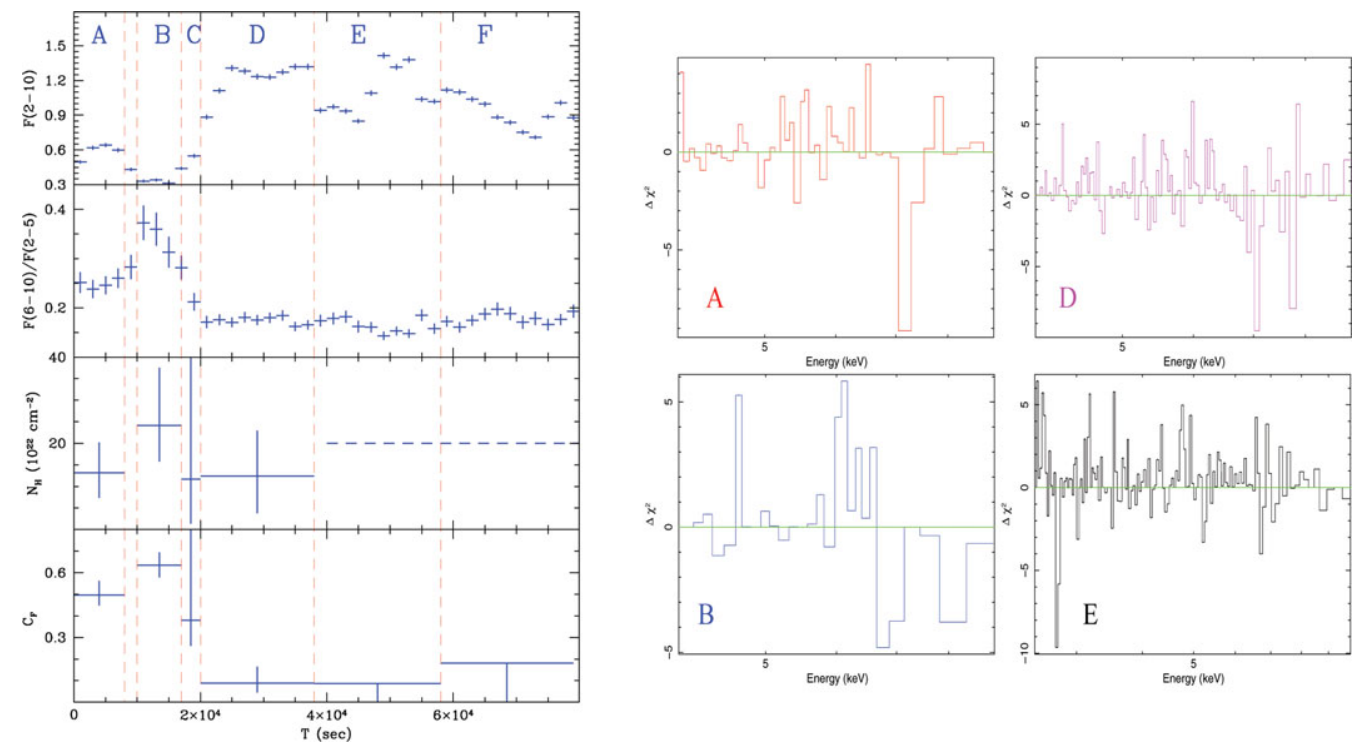

Figure 5. Left: same as in Figure 3, for the second orbit of the XMM-Newton observation of Mrk 766. Right: residuals for the intervals indicated in the left panel. The presence of iron absorption lines suggests the presence of an ionized tail.

be improved by relaxing the assumption of constant $N_{\mathrm{H}}$. In doing so, in most of the cases analyzed so far, we find that it is impossible to significantly constrain the values of $N_{\mathrm{H}}$ and $C_{\mathrm{F}}$ during the different phases of an eclipse due to their strong degeneracy. In other words, it is only possible to study the evolution of one parameter, freezing the other one. The only exceptions found so far, i.e. spectra with enough signal-to-noise to analyze the evolution of the two parameters at the same time, are Mrk 766 and NGC 1365.

In Figure 4a, we show the results of a recent analysis of a Suzaku observation of NGC 1365 (Maiolino et al. 2010, submitted), where the hardness ratio light curve clearly shows at least two possible occultation events. We performed a complete spectral analysis of several short intervals before, during and after the eclipses, and we obtained the results shown in the two lower panels of Figure 4, showing the light curves of the covering factor and the column density. The most interesting results come from the second eclipse: the covering factor $C_{\mathrm{F}}$ increases during the occultation phase, with a constant $N_{\mathrm{H}} \sim 3 \times 10^{23} \mathrm{~cm}^{-2}$. Then, in the subsequent phase, $C_{\mathrm{F}}$ remains compatible with unity (i.e., complete covering) while the column density decreases. This behavior can only be explained by a "cometary shape" of the obscuring cloud, with a high column density head, and a tail with decreasing $N_{\mathrm{H}}$.

An analogous result has been obtained (though with smaller statistical significance) for the first two eclipses in Mrk 766 (Figure 4B). The third eclipse of Mrk 766 is instead too fast to allow a detailed spectral analysis (the spectral counts in such short time intervals are not enough to break the degeneracy between $C_{\mathrm{F}}$ and $N_{\mathrm{H}}$, as shown in Figure 5). However, a strong indication of a cometary tail comes from the residuals with respect to the best fit model during and after the eclipse, showing strong absorption lines due to FexxV in outflow with a velocity of $10-15000 \mathrm{~km} \mathrm{~s}^{-1}$. These features completely disappear after $\sim 40 \mathrm{ks}$. A complete analysis of these spectra, where we demonstrate the high statistical significance of the line detections, is presented in a dedicated paper (Risaliti et al. 2010, submitted). The highly ionized component revealed by the absorption lines 
Table 1. List of Sources with $N_{\mathrm{H}}$ Variations on Short Time Scales

\begin{tabular}{l|c|c|c|c}
\hline Name & $\Delta\left(N_{\mathrm{H}}\right)^{a}$ & $\Delta(T)^{b}$ & Method $^{c}$ & Ref. \\
\hline NGC 1365 & $>10^{24}$ & $<2$ days & Snapshot & 1,2 \\
NGC 1365 & $3 \times 10^{23}$ & 10 hours & Continuous & 3,4 \\
NGC 4388 & $2 \times 10^{23}$ & 15 hours & Continuous & 1 \\
NGC 4151 & $2 \times 10^{23}$ & 20 hours & Continuous & 5 \\
NGC 4151 & $10^{23}$ & $<2$ days & Snapshot & 1 \\
NGC 7582 & $10^{23}$ & 20 hours & Snapshot & 6 \\
Mrk 766 & $3 \times 10^{23}$ & 10 to 20 hours & Continuous & 1,7 \\
MCG-6-30-15 & $10^{23}$ & 10 hours & Continuous & 1 \\
UGC 4203 & $3 \times 10^{23}$ & $<15$ days & Snapshot & 1 \\
NGC 3227 & $7 \times 10^{22}$ & 1 day & Continuous & 8 \\
\hline
\end{tabular}

Notes: ${ }^{a} N_{\mathrm{H}}$ variations in $\mathrm{cm}^{-2} ;{ }^{b}$ duration of the observed eclipse; ${ }^{c}$ Observational method: repeated snapshot observations, or analysis of long continuous observations. References: 1: this work; 2: Maiolino et al. (2010), submitted; 3: Risaliti et al. (2007); 4: Risaliti et al. (2009); 5: Puccetti et al. (2007); 6: Bianchi et al. (2009); 7 : Risaliti et al.(2010), submitted.; 8: Liu et al.(2010), in preparation

strongly suggests the presence of a ionized outflowing tail associated with the obscuring cloud.

We note that this highly ionized component is not present in the first two occultation events in Mrk766, but only in the third, much faster event. Even if one case is clearly not enough to build a complete model, the data suggest a scenario where the obscuring clouds are distributed in a large range of distances from the center (if we assume Keplerian velocity, the factor $\sim 4-5$ of spread in velocities corresponds to a factor of $\sim 20$ of spread in distances), and where the clouds closer to the center are also the more ionized ones. This simple scheme is illustrated in Figure 6.

\section{Conclusions and Future Work}

We have presented new time-resolved spectral studies of several bright AGNs, showing column density variability on time scales of a few hours to a few days. The main results of our analysis are:

1. Fast (hours to days) column density variability is common among AGNs. This implies that the observed variable X-ray absorption is due to clouds with velocities, densities, sizes and distances from the central black hole of the same order of those of BLR clouds.

2. In the highest signal-to-noise studies, it is possible to investigate the structure and shape of single obscuring clouds in detail. This reveals a "cometary" profile, with a high column-density head and a tail with decreasing $N_{\mathrm{H}}$.

These results show that the X-ray absorption is at least in part due to BLR clouds, and that X-ray spectroscopy can be a powerful tool to directly measure the physical properties of the broad-line region in AGNs.

The work presented here is only the first part of an on-going comprehensive analysis of all the bright AGNs with long high-quality X-ray observations. At present, the evidence of "common" short timescale $N_{\mathrm{H}}$ variations presented here is based on a sparse sample of sources, with no homogeneous selection criteria. The next major step is therefore the selection and the homogeneous analysis of a representative sample of the local AGN population. This work will provide a quantitative estimate of the occurrence of $N_{\mathrm{H}}$ variability on short timescales, and hopefully will lead to the discovery of more high signal-to-noise spectra of occultations, such as those found in NGC 1365 and Mrk 766, in order to perform other studies of the physical properties of BLR clouds. 


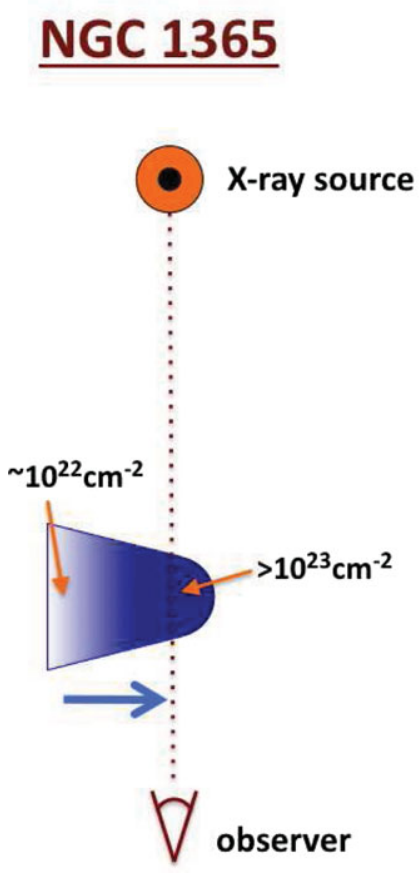

\section{MKN 766}

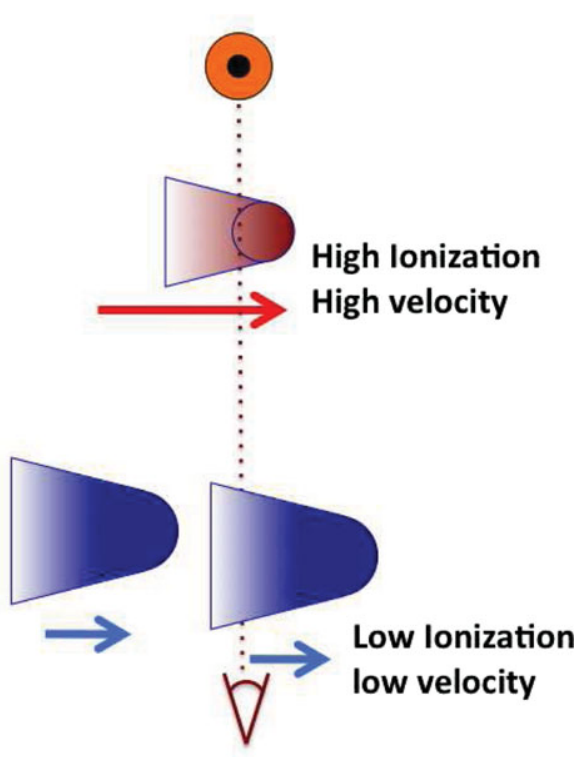

Figure 6. A schematic view of the X-ray absorbing clouds, as estimated from the observations described here. The eclipsing cloud in NGC 1365 is the one with the best-measured properties, with a column density going from $\sim 3 \times 10^{23} \mathrm{~cm}^{-2}$ in the head, to a few $10^{22} \mathrm{~cm}^{-2}$ in the tail (Maiolino et al. 2010). In Mrk 766, we suggest that the fast cloud with the ionized tail is much closer to the center than the two slower, neutral clouds. The ionized tail in the fast cloud is probably in outflow (not shown here; a complete analysis is presented by Risaliti et al. 2010).

\section{Acknowledgements}

This work has been partially supported by NASA grants NNX08AX78G and G089107X, and by grant ASII-INAF I/088/06/0.

\section{References}

Bianchi, S., Piconcelli, E., Chiaberge, M., Bailón, E. J., Matt, G., \& Fiore, F. 2009, ApJ, 695, 781

Elvis, M., Risaliti, G., Nicastro, F., Miller, J. M., Fiore, F., \& Puccetti, S. 2004, ApJ, 615, L25

Miller, L., Turner, T. J., Reeves, J. N., George, I. M., Kraemer, S. B., \& Wingert, B. 2007, $A \mathscr{E} A, 463,131$

Puccetti, S., Fiore, F., Risaliti, G., Capalbi, M., Elvis, M., \& Nicastro, F. 2007, MNRAS, 377, 607

Risaliti, G., Elvis, M., \& Nicastro, F. 2002, ApJ, 571, 234

Risaliti, G., Elvis, M., Fabbiano, G., Baldi, A., \& Zezas, A. 2005, ApJ, 623, L93

Risaliti, G., Elvis, M., Fabbiano, G., Baldi, A., Zezas, A., \& Salvati, M. 2007, ApJ, 659, L111

Risaliti, G., et al. 2009, ApJ, 696, 160

Turner, T. J., Miller, L., Reeves, J. N., \& Kraemer, S. B. 2007, A\& A, 475, 121 